\title{
Hydatid cyst of the orbit in a young Nigerian female: a case report
}

\author{
Oluyemi Fasina ${ }^{1}$ and Olabiyi G. Ogun ${ }^{2}$ \\ Ghana Med J 2017; 51(4):204-206 DOI: http://dx.doi.org/10.4314/gmj.v51i4.11
}

${ }^{1}$ Oculoplastics and Ocular Oncology Unit, Department of Ophthalmology, University of Ibadan/University College Hospital, Ibadan, Oyo State, Nigeria. ${ }^{2}$ Pathology Department, University of Ibadan/University College Hospital, Ibadan, Oyo State, Nigeria.

Corresponding author: Dr. Oluyemi Fasina

E-mail: yemifash2000@yahoo.com

Conflict of interest: None declared

\section{SUMMARY}

Objective: To report an unusual case of orbital hydatid cyst successfully managed by surgical excision.

Case report: A 33-year-old female presented with gradual protrusion of the left eye associated with poor vision, cosmetic embarrassment and watering. She had excision biopsy of an encapsulated cystic lesion with cheesy tissue and serous content. Orbital hydatid cyst due to echinococcosis was confirmed at histopathological examination of the excised tissue. She subsequently became pregnant hence, could not undergo post-operative antihelminthic treatment, but was managed conservatively with no recurrence after five years of regular follow-up.

Conclusion: Orbital hydatid cyst should be considered as a differential in a young patient presenting with unilateral proptosis

Funding: None

Keywords: Echinococcosis, Hydatid cyst, Orbit, Nigerian, Proptosis

\section{INTRODUCTION}

Hydatid disease is one of the zoonotic diseases of public health importance. ${ }^{1}$ It is a chronic cyst-forming parasitic helminthic disease affecting human beings, domestic, and wild ungulates. ${ }^{2}$ Of the two presentations of the disease with medical importance, cystic echinococcosis caused by the larval form of dog tape worm, Echinococcus granulosus, and alveolar echinococcosis, caused by Echinococcus multilocularis, the former, accounts for about $95 \%$ of clinical presentation of the disease in humans. ${ }^{3}$ It has a worldwide geographical distribution but more common in temperate countries and the sheep strain is responsible for most human infections. ${ }^{3}$ Hydatidosis occurs in humans with varying morbidity and mortality following invasion of various organs by the larval form of the parasite in the primary infective disease. ${ }^{4}$ There are presently no reports of hydatid cyst affecting the orbit in the country, to the best of the authors' knowledge, hence, this reported case in an adult Nigerian female.

\section{CASE REPORT}

A 33-year-old female presented to the Eye Clinic with history of protrusion of the left eye, associated with poor vision, cosmetic embarrassment and watering.

She had an existing record of being managed in our facility 22 years earlier with clinical history of painless protrusion of the same eye, however, vision was normal, and no palpable orbital mass detected then. She had surgical biopsy of a deep orbital mass after plain radiographs and B-scan ultrasonography showed illdefined orbital soft tissue mass. Histopathological examination report of the biopsied tissue then was suggestive of non-specific orbital inflammatory disease for which she was treated with oral prednisolone. She however, abandoned her treatment and re-presented with gradual protrusion of the eye associated with poor vision. Ocular examination at present consultation revealed acuity of counting fingers at 2 meters in the left eye, relative afferent pupillary defect, non-axial proptosis of about $20 \mathrm{~mm}$, palpable soft tissue deep orbital mass mainly in the superior orbit. (Figure 1a). There was mild tenderness on palpation, retropulsion of the globe, but no palpable thrill or bruit. Fundoscopy showed diffuse disc pallor. Orbital computed tomography (CT) scan showed an isodense retrobulbar cystic mass with a rim of calcification in some areas and a central nidus of roundish amorphous calcification laterally. (Figure 1b and 1c).

An impression of multiloculated retrobulbar cystic mass, was made and she had orbitotomy with excision of the lesion. Intra-operatively, a cystic mass with cheesy tissue and serous content was excised. 


\section{Case Report}

There was however, rupture of the cyst capsule with spillage of the serous content into the orbit and, thorough saline irrigation of the orbit was done.

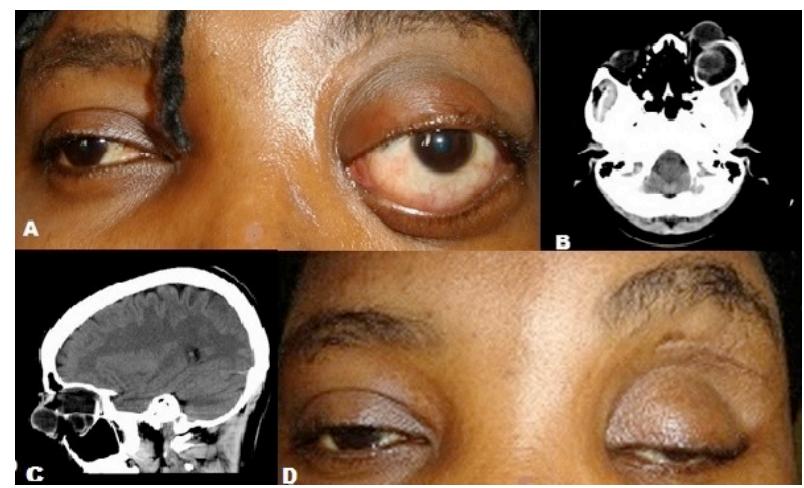

Figure 1 Clinical and CT scan pictures of the patient

A: Pre-operative clinical picture of the patient showing left non-axial proptosis

B: CT scan (axial cut) picture of the patient showing the isodense cystic mass

C: CT scan (sagittal cut) of the patient showing the retrobulbar mass and a loculated mass within it

D: Post-operative clinical picture of the patient with severe ptosis

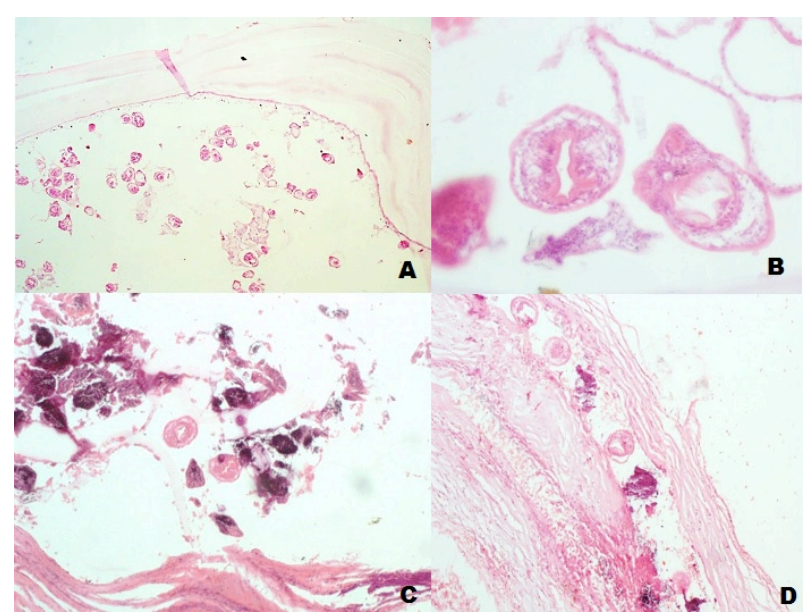

Figure 2 Photomicrograph of the histology slides of the patient

A: Low power magnification of the germinal epithelium of the hydatid cysts giving off brood capsules and protoscoleses $(\mathrm{x} 40)$

B: Higher power magnification showing details of the protoscolex with hooks and suckers (x400)

$\mathrm{C}$ and D: Show dystrophic calcification of proscoleses and stromal fibrosis (x100)

Histopathological examination of the specimen showed a cyst with 2 layered laminated wall with a lining germinal epithelium. Figure $2 \mathrm{a}$ shows the epithelium giving up brood capsules of Echinococcus granulosus with multiple protoscoleses, and a detailed morphology of the protoscolex with hooks and suckers is shown in
Figure 2b. Focal areas of rupture with resultant foreign body type granulomas, stromal fibrosis and extensive dystrophic calcification of non-viable protoscolex are observed (Figures 2c and 2d).

She was planned to receive post-operative oral Albendazole $15 \mathrm{mg} / \mathrm{Kg}$ and Prednesolone $1 \mathrm{mg} / \mathrm{Kg}$, but before commencement of chemotherapy, she became pregnant and was thus placed on observation and regular follow-up. Following five years of regular follow-up, there had been no recurrence, and she has been counseled to have levator aponeurotic reattachment surgery to correct her post-operative ptosis. (Figure 1d)

\section{DISCUSSION}

Echinococcosis presents with varied clinical manifestation and disease severity in man, from an asymptomatic disease to a fatal one. ${ }^{4}$ Symptomatology in cystic echinococcosis is usually due to the resultant pressure effect on the surrounding tissues or rupture of the cyst, and this is dependent on the organ affected, and, the size and position of the cyst within the organ. ${ }^{1}$ Although any age group can be affected, most patients with liver and lung infections usually become symptomatic at adulthood due to the slow development of the cyst, reportedly at about $1.5 \mathrm{~cm}$ annually. ${ }^{5}$

However, due to the limited space within the orbit, most cases of orbital hydatid disease are usually diagnosed early at childhood. ${ }^{6}$ Our patient presented at adulthood, possibly due to the previous orbital surgery that could have altered the tissue plane and space within her orbit. The dystrophic calcification noted on the CT scan and on histology also suggests that the lesion had been present for some considerable period of time. Cystic echinococcosis commonly affects the liver and lungs in over $90 \%$ of cases while other organs affected include the mesentery, abdominal wall, spleen, and rarely, the bronchus, parotid, uterus, tibia and foot. ${ }^{1,6-8}$ The orbits are rarely involved in echinococcosis, accounting for about $1 \%$ of all systemic locations ${ }^{7,9}$

The most common presentation is proptosis due to the sp.ace-occupying nature of the cyst while other clinical manifestations include chemosis, ptosis, lid edema, restriction of extraocular motility and visual disturbance. ${ }^{9}$ Also, long standing cases as in our patient, can result in compressive optic neuropathy and optic atrophy. ${ }^{8}$

In endemic areas, diagnosis of orbital hydatid cyst is mostly clinical, and, positive serological tests, plain radiographs, and history of infection in other parts of the body with E. granulosus aided the diagnosis while confirmation is made at surgery. ${ }^{9}$ 
Recently, imaging studies like Computed Tomography scan and ultrasonography had improved the preoperative diagnosis of the disease by demonstrating single or multiple cystic lesion with well-defined borders. $^{10}$ Although imaging studies are not confirmatory of the disease, they assist in planning the surgical approach to manage the cases ${ }^{5}$ and, in our patient, a cystic mass was noted in the superior orbit following which she had cyst excision through superior orbitotomy.

Confirmation of hydatid disease is usually by direct identification of the typical protoscoleces or hooks of E. granulosus in aspirates from the hydatid cysts or histological examination of biopsied tissue specimen. ${ }^{8}$ Histopathological examination of the excised cyst capsule in our patient confirmed echinococcosis by identifying multiple protoscoleses in the germinal epithelial layer of the cystic wall.

The main treatment for orbital echinococcosis is surgery ${ }^{8}$ and different surgical approaches had been reported in literature ${ }^{7,9,11}$ depending on the site and size of the cyst, and, surgeon's preference and expertise. ${ }^{7}$ A major complication of surgical removal of the cyst however, is intra-operative rupture of the cyst with associated risk of recurrence and secondary infection. ${ }^{10}$ The cystic capsule in our patient ruptured intraoperatively, and thorough saline irrigation of the orbit was done.

Chemotherapeutic agents used in treatment of hydatid cyst presently include albendazole, a benzimidazole antihelminthic used either as a non-surgical intervention for orbital echinococcosis, or as part of the general pre/post-operative management, administered as monotherapy, or in combination with praziquantel. $8,9,12$ The teratogenic effects of albendazole however, had been reported in animals, and although its use is not absolutely contraindicated in pregnancy, caution and specialists' advice are advocated. ${ }^{1}$

Our patient became pregnant shortly after histopathological confirmation of echinococcosis hence, she was not treated post-operatively with oral albendazole due to the potential teratogenic effect.

She was managed conservatively with regular follow-up clinic visits. Noteworthy is that she had no recurrent or secondary infection after a 5-year follow-up period despite the rupture of the hydatid cyst at surgery. She is presently awaiting corrective surgery for post-operative aponeurotic ptosis in the country.

\section{CONCLUSION}

Echinococcosis affecting the orbit is rare and not previously reported in Nigeria. It should, however, be considered a differential in unilateral proptosis..

\section{REFERENCES}

1. McManus DP, Zhang W, Li J, Bartley PB. Echinococcosis. Lancet. 2003;362:1295-304

2. Craig PS, McManus DP, Lightowlers MW, Chabalgoity JA, Garcia HH, Gavidia CM, et al. Prevention and control of cystic echinococcosis. Lancet Infect Dis. 2007;7:385-94

3. Budke CM, Deplazes P, Torgerson PR. Global socioeconomic impact of cystic echinococcosis. Emerg Infect Dis. 2006;12:296-303

4. Eckert J, Deplazes P. Biological, Epidemiological, and Clinical Aspects of Echinococcosis, a Zoonosis of Increasing Concern. Clin Microbiol Rev. 2004;17(1):107-35

5. Gomez MA, Croxatto JO, Crovetto L, Ebner LR. Hydatid Cysts of the Orbit. A Review of 35 Cases. Ophthalmology. 1988;95:1027-32

6. Moro P, Schantz PM. Echinococcosis: a review. Int J Infect Dis. 2009;13:125-33

7. Ciurea AV, Giuseppe G, Machinis TG, Coman TC, Fountas KN. Orbital hydatid cyst in childhood: a report of two cases. South Med J. 2006;99(6):620-4

8. Xiao A, Xueyi C. Hydatid cysts of the orbit in Xinjiang: a review of 18 cases. Orbit. 1999;18(3):151-5

9. Ghosh A, Saha S, Jha A, Chandra S. Orbital hydatid cyst- A rare case report. Indian J Otolaryngol Head Neck Surg. 2008;60:191-3

10. Turgut AT, Altin L, Topcu S, Kilicoglu B, Altinok $\mathrm{T}$, Kaptanoglu E, et al. Unusual imaging characteristics of complicated hydatid disease. Eur $J$ Radiol. 2007;63:84-93

11. Kahveci R, Sanli AM, Gürer B, Sekerci Z. Orbital hydatid cyst. J Neurosurg Pediatr. 2012;9:42-4

12. Sihota R, Sharma T. Albendazole therapy for a recurrent orbital hydatid cyst. Indian J Ophthalmol. 2000;48(2):142-3త 